\title{
Hepatic Tumor as Antenatal Presentation of Costello Syndrome
}

\author{
Chusana Petpichetchian, M.D. ${ }^{1}$, Richard Brown, M.D., MBBS, FRCOG, FACOG ${ }^{2}$, \\ Gabriel Altit, M.D., C.M., FRCPC, FAAP ${ }^{3}$, Karl Muchantef, M.D. ${ }^{4}$, \\ Isabelle De Bie, M.D., Ph.D., FRCPC, FCCMG ${ }^{5}$
}

'Department of Obstetrics and Gynecology, Faculty of Medicine, Prince of Songkla University, Hat Yai, Songkhla 90110, Thailand.

${ }^{2}$ Department of Obstetrics and Gynecology, McGill University Health Centre, Montréal, QC H4A 3J1, Canada. ${ }^{3}$ Division of Neonatology, Department of Pediatrics, McGill University Health Centre, Montréal, QC H4A 3J1, Canada. ${ }^{4}$ Department of Radiology, McGill University Health Centre, Montréal, QC H4A 3J1, Canada.

${ }^{5}$ Division of Medical Genetics, Department of Specialized Medicine, McGill University Health Centre, Montréal, QC H4A 3J1, Canada.

Received 3 June 2020 • Revised 8 July 2020 • Accepted 18 July 2020 • Published online 17 November 2020

\begin{abstract}
:
A large hepatic mixed echoic mass occupying the left fetal abdomen was identified at $26^{6 / 7}$ weeks. The mass showed peripheral and internal vascularity. Other ultrasound findings included edema of the fetal head and face, macrosomia, shortened long bones, abnormal posture of hands, small stomach, polyhydramnios and biventricular hypertrophy. Fetal magnetic resonance imaging confirmed a hypervascular mass replacing the lateral left hepatic lobe, suggestive of a congenital hemangioma. The fetus was delivered by cesarean section at $28^{2 / 7}$ weeks. The baby was stabilized at day 3 of life, and underwent successful selective tumor embolization. The baby remained stable for 3 days, then deteriorated with a progressive thickening of the myocardium. The child then passed away on day 11 from severe progressive hypertrophic cardiomyopathy, with almost complete obliteration of the left ventricular cavity; an autopsy was declined. Postnatal investigations reported a de novo heterozygous pathogenic HRAS variant (NM_005343.3(HRAS): c.35_36 delinsTT, p.Gly21Val), previously reported in 8 cases associated with the early, lethal form of Costello syndrome.
\end{abstract}

Keywords: Costello syndrome, hemangioma, hepatic tumor, Rasopathies, ultrasound

Contact: Isabelle De Bie, M.D., Ph.D, FRCPC, FCCMG Division of Medical Genetics, Department of Specialized Medicine, McGill University Health Centre, Montréal, QC H4A 3J1, Canada.

E-mail: isabelle.debie@muhc.mcgill.ca

. Hesting by Prince of Songkla University. All rights reserved.

This is an open access article under the CC BY-NC-ND license

(http://www.jhsmr.org/index.php/jhsmr/about/editorialPolicies\#openAccessPolicy). 


\section{Introduction}

Costello syndrome (CS) is a rare genetic condition, in part of the spectrum of disorders called Rasopathies (best known as Noonan-spectrum disorders). CS results from germline variations in the gene HRAS. Common postnatal features of CS include: failure to thrive, cardiac involvement, hypotonia, as well as some degree of developmental delay. ${ }^{1}$ Individuals with CS also present with an increased risk of malignant tumors, particularly in childhood, with a cumulative incidence of $15.0 \%$ by the age of $20 .^{1,2}$

Prenatal diagnosis of CS can be challenging, because ultrasound (US) findings associated with this condition are non-specific. The most common prenatal feature reported in association with CS is polyhydramnios. Other findings include: nuchal thickening, ascites, hydrops, a small or absent stomach, short long bones, ulnar deviation of the wrists, macrosomia, macrocephaly, hepatomegaly, cardiomyopathy, and tachycardia. ${ }^{1,3-9}$

The p.Gly12Val (NM_005343.3(HRAS):c.35_36 delinsTT) pathogenic variant is a recurring CS variant that has been associated with an early, lethal form of this condition. ${ }^{8}$ To date, 8 cases of CS caused by this particular variant have been reported, all presenting with lethal outcomes. $^{4,5,9,10}$ This report is the ninth case, with initial fetal presentation of hepatic tumor, which has never previously been described as an antenatal manifestation of CS.

\section{Case report}

This report describes the third pregnancy of a 37-year-old woman, with an unremarkable prior obstetrical history. Her first trimester US, including nuchal translucency (NT) evaluation, was normal. Integrated NT and biochemical aneuploidy screening was performed, and yielded a low risk result; despite serum markers notable for elevated human chorionic gonadotropin levels at 4.67 multiples of median (MoM), alpha-fetoprotein levels at 2.53 MoM, and inhibinA levels at 4.74 MoM. Fetal anatomy survey at 21 weeks of gestation was reported normal. A second fetal ultrasound at $26^{6 / 7}$ weeks of gestation identified a large mixed echogenic mass measuring 60x $57 \times 49 \mathrm{~mm}$ occupying the left fetal abdomen (Figure 1). The mass appeared well demarcated from the surrounding structures, except where it abutted against the left hepatic lobe, suggesting that it arose from the liver. The mass was vascular with an arterial origin and venous return that appeared confluent with the left-sided hepatic vessels. Other sonographic findings included severe skin edema of the head and face (Figure 2), macrosomia, shortened long bones, abnormal posture of hands (Figure 2), small stomach, thick placenta, polyhydramnios with an amniotic fluid index of $47 \mathrm{~cm}$, and reversal of end-diastolic flow in the umbilical arteries on Doppler evaluation, compatible with a steal effect from the abdominal aorta by the lesion with high vascularity. Although the cardiac structure and contractility appeared normal, regurgitation at both atrioventricular valves was noted. Fetal echocardiogram reported mild biventricular hypertrophy. There was no ascites, pleural effusion or other evidence of hydrops noted.

Fetal magnetic resonance imaging (MRI) showed a mass replacing the lateral left hepatic lobe. The hepatic artery was markedly enlarged, as was the left hepatic vein, confirming a hypervascular lesion. Based on both US and MRI results, the lesion was thought to represent a large hemangioma, most likely a rapidly involuting congenital hemangioma. Hepatoblastoma and mesenchymal harmartoma were considered as well, but were felt to be less likely. 


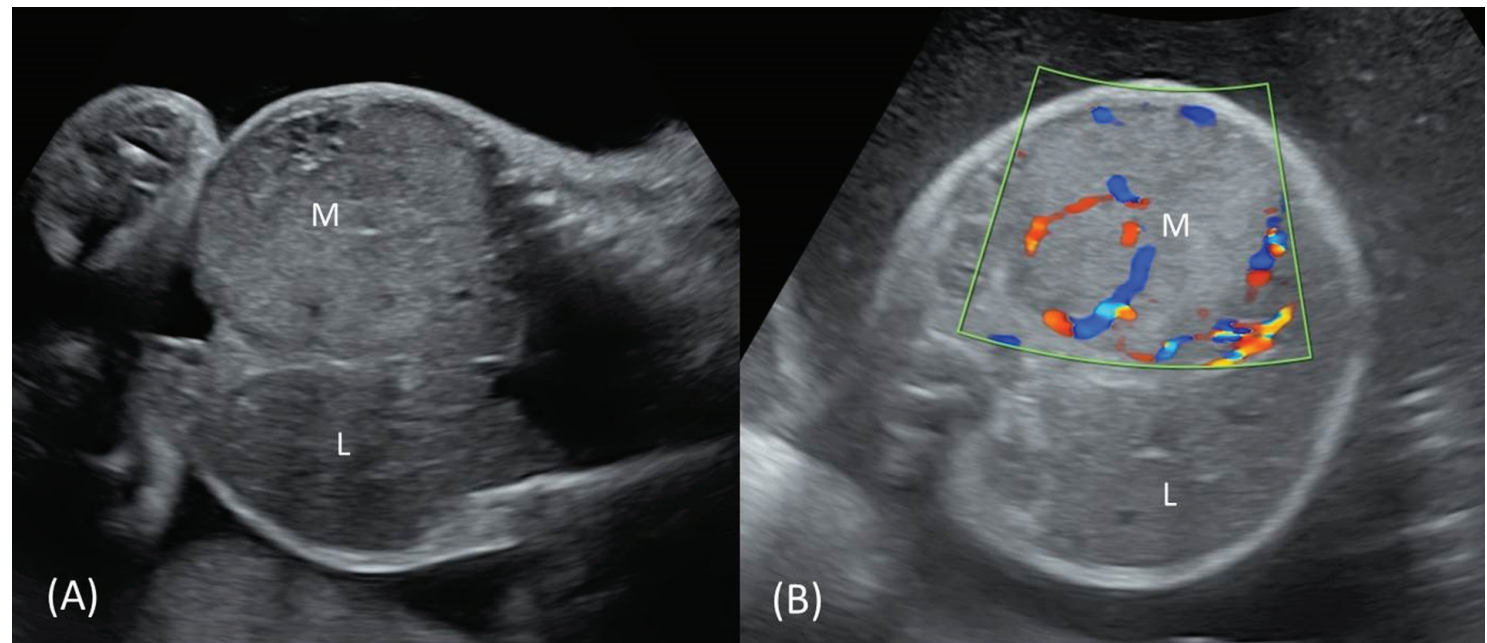

$M=$ mass, $L=$ liver

Figure 1 Fetal hepatic mass. (A) Coronal view of fetal abdomen demonstrating a large mass occupying the left side of the abdomen, extending from the level of diaphragm to the pelvis. (B) Axial view of fetal abdomen with color-Doppler imaging, demonstrating increased vascularity at the periphery and within the mass

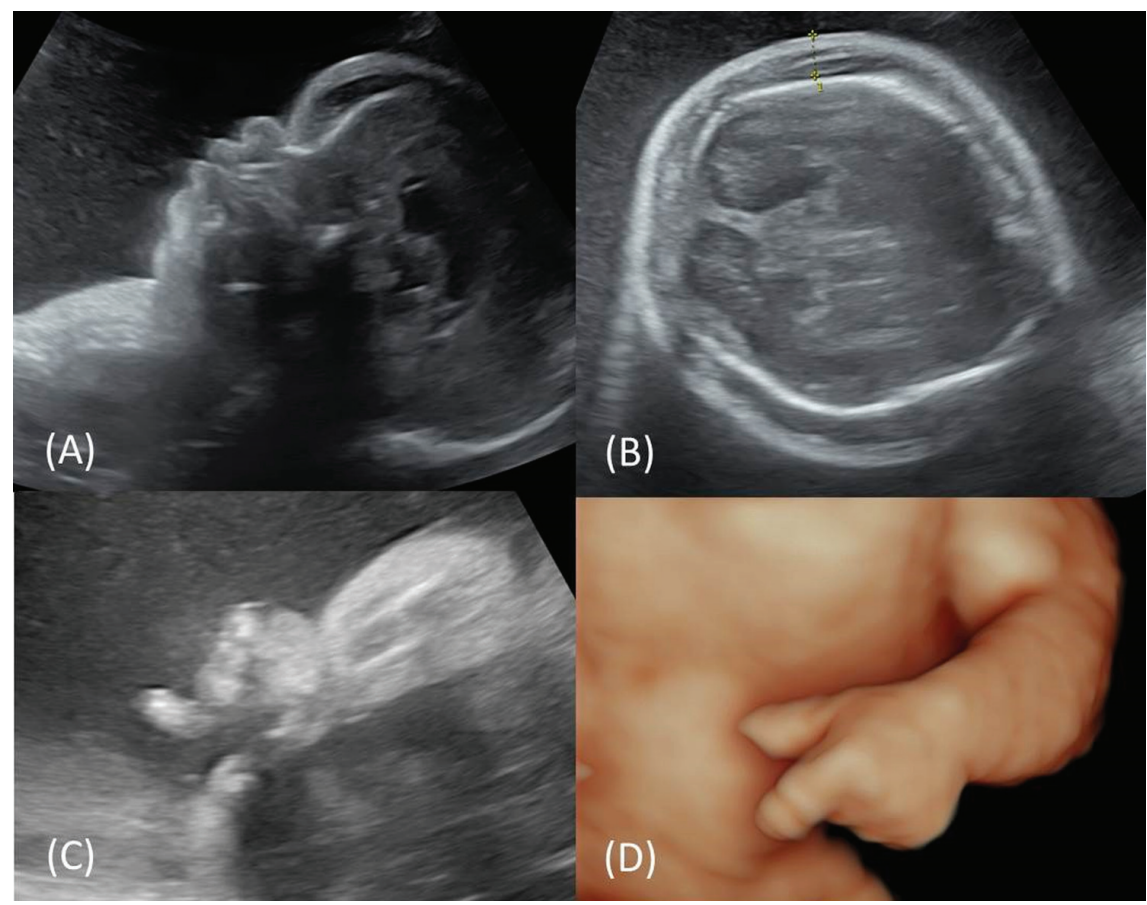

Figure 2 Associated US findings in the fetus. (A) Fetal profile view, demonstrating subcutaneous edema of the scalp and facial areas. (B) Axial view of the fetal head. The scalp thickness measured $9.4 \mathrm{~mm}$. (C, D) Fetal hands fixed in abnormal posture 
The patient was admitted for close fetal surveillance and administration of corticosteroids. A multidisciplinary team, including, maternal-fetal medicine specialists, pediatric surgeons, medical geneticists, intervention radiologists and neonatologists, was involved in planning the care of the pregnancy. Amniocentesis with amnioreduction was discussed, but the patient developed irregular uterine contractions. Concerted decision with the parents for an expectant management approach with frequent fetal health evaluation was therefore adopted.

After 10 days of observation, the fetal condition deteriorated with a non-reassuring fetal heart rate tracing, prompting an emergency cesarean section at the gestational age of $28^{2 / 7}$ weeks. Birthweight was 1,800 grams (above $99^{\text {th }}$ percentile for gestational age). Physical examination of the newborn was notable for marked skin edema, which limited evaluation of the facial features, and a distended abdomen. Echocardiography demonstrated biventricular hypertrophy. A postnatal ultrasound was performed and confirmed the presence of a large mass replacing the lateral left lobe of the liver, measuring $5.7 \times 4.3 \times 6.6 \mathrm{~cm}$. The lesion was heterogeneous in texture with some anechoic areas and was hyper-vascular, with low resistance arterial and phasic venous waveforms. There was no internal calcification. The celiac and hepatic arteries were enlarged, and showed low resistance waveforms. This was consistent with a diagnosis of congenital hepatic hemangioma. No MRI was repeated in the postnatal context, due to the cardio-respiratory instability of the newborn.

A multi-disciplinary approach was coordinated between neonatology, interventional radiology, surgery, dermatology and haematology-oncology. The parents were involved throughout the entire decision process and informed about the risks, prognosis and clinical concerns. Therapy was tailored to the parents' wishes and expectations. On post-natal day 1 the newborn was started on systemic steroids, due to significant cardio- respiratory instability. The baby presented signs of consumptive coagulopathy and required inotropic support, as well as significant positive mean airway pressure ventilation to maintain adequate oxygenation. Despite successive transfusions, coagulation and haematological profiles remained consistently disturbed and the baby showed clinical signs of end-organ hypoperfusion, as well as pulmonary hypertension. The baby was medically stabilized over the course of the first 72 hours of life, in order to avoid the time window associated with a significant increased risk of intra-ventricular haemorrhage. Concerns over persistent cardio-respiratory instability as well as significant burden of medical therapy to achieve homeostasis in the context of prematurity, led to the decision to attempt embolization in the hopes to wean some of the supportive therapy and accelerate the hepatic mass involution.

Selective embolization of the tumor was performed through the umbilical artery. Initial angiographic images demonstrated marked arterio-venous shunting. Three of the four feeding vessels to the lesion could be catheterized. These three vessels were embolized with coils. The fourth feeding was identified, but was left intact to avoid excessive hemodynamic shifts. The post-embolization angiogram demonstrated markedly decreased flow and shunting through the lesion. Post-embolization, the newborn remained stable for 3 days. While there was initial improvement in the hemodynamics and hematological condition, the newborn deteriorated at day 7 of life, with a progressive thickening of the myocardium on echocardiography. Despite aggressive efforts to stabilize the baby, the patient passed away on day 11 from severe progressive hypertrophic cardiomyopathy, with almost complete obliteration of the left ventricular cavity; an autopsy was declined.

Diagnostic investigations, undertaken during the neonatal period, reported a de novo heterozygous pathogenic HRAS variant (NM_005343.3(HRAS):c.35_36 delinsTT, p.Gly21Val), previously reported to be associated 
with the early, lethal form of CS. Both parents were tested for the familial pathogenic variant, and found not to be carriers.

\section{Discussion}

This report is the first of a hepatic mass as an antenatal presentation of CS.

The majority of prenatal CS reports describe ultrasound findings in the second trimester, with polyhydramnios being the most common presentation, in combination with a variety of other nonspecific findings. Of the only two fetal CS cases with molecular confirmation, the predominant findings were of macrosomia and polyhydramnios. In the first one, three-dimensional (3D) imaging made possible detection of "coarse" facial features (macrocrania, wide nasal bridge, low-set ears, puffy cheeks); although in this case, an overgrowth condition (Sotos syndrome) was suspected first. ${ }^{6}$ Interestingly, the second case initially presented with abnormal serum markers, with a positive quadruple serum screen (specific marker values not reported), and nuchal edema, macrosomia, shortened limbs, ventriculomegaly, and polyhydramnios in the second trimester, which led to the suspicion of CS. ${ }^{11}$

In the present case, the findings of a hepatic tumor, macrosomia and polyhydramnios were initially suggestive of an overgrowth condition such as Beckwith-Wiedemann syndrome, which was considered in the differential diagnosis, along with Noonan spectrum disorders (this diagnosis having been considered on the basis of fetal skin edema, ventricular hypertrophy and polyhydramnios). Postnatal features were more consistent with a Rasopathy spectrum disorder as a possible aetiology, which was confirmed by subsequent molecular investigations.

The prenatal findings of this case are consistent with the more frequently reported US features of CS, namely polyhydramnios, macrosomia, and shortened long bones. The p.Gly12Val HRAS pathogenic variant has been associated with a lethal outcome in all CS cases reported so far. This emphasizes the importance of timely prenatal genetic diagnosis in the planning of pregnancy care and neonatal management.

CS is associated with an increased risk of malignancy, the most prevalent being rhabdomyosarcoma and neuroblastoma, both embryonal tumors that are also prevalent in Beckwith-Wiedemann syndrome., ${ }^{2,12}$ Hepatomegaly has been reported in fetuses with CS, but our case is the first to present with a distinct hepatic tumor. One limitation of this study is that the definitive histological diagnosis of the tumor could not be obtained as an autopsy was declined. Prenatal imaging findings were most suggestive of hemangioma, and angiography findings also supported this. This finding is felt to be part of the phenotypic expression of CS as vascular proliferative lesions have previously been reported in association with HRAS vatiants.

CS results from de novo pathogenic variants in the gene HRAS. ${ }^{1}$ In the current case, both parents were tested for the familial pathogenic variant, and were found not to be carriers. Therefore, the risk of recurrence for this couple is estimated to be less than $1.0 \%$, as the possibility of gonadal mosaicism can never be completely excluded.

\section{Conclusion}

We report the case of a fetus affected with a rare and lethal form of CS, associated with the pathogenic HRAS p.Gly12Val variant, that presented prenatally with a large hepatic tumor, polyhydramnios, macrosomia, shortened long bones, abnormal hand posture, and small stomach. CS should be considered when polyhydramnios is encountered in combination with other suggestive findings, or in situations suggesting fetal overgrowth. The use of 3D US may be helpful to identify characteristic facial features. Timely use of invasive diagnostic testing has important implications on management of the pregnancy and newborn. 


\section{References}

1. Gripp KW, Rauen KA. Costello syndrome. In: Adam MP, Ardinger HH, Pagon RA, Wallace SE, Bean LJH, Stephens K, et al, editors. GeneReviews ${ }^{\circledR}$ [monograph on the Internet]. Seattle (WA): University of Washington; 1993-2020 [cited 2019 Aug 29]. Available from: https://www.ncbi.nlm.nih.gov/books/ NBK1507/

2. Kratz CP, Rapisuwon S, Reed H, Hasle H, Rosenberg PS. Cancer in Noonan, Costello, cardiofaciocutaneous and LEOPARD syndromes. Am J Med Genet C Semin Med Genet 2011;157C:83-9.

3. Lin AE, O'Brien B, Demmer LA, Almeda KK, Blanco CL, Glasow $\mathrm{PF}$, et al. Prenatal features of Costello syndrome: ultrasonographic findings and atrial tachycardia. Prenat Diagn 2009; 29:682-90

4. Quelin C, Loget P, Rozel C, D'Herve D, Fradin M, Demurger $\mathrm{F}$, et al. Fetal costello syndrome with neuromuscular spindles excess and p.Gly12Val HRAS mutation. Eur J Med Genet 2017;60:395-8.

5. Bend EG, Louie RJ, Stevenson RE. Fetal edema, not overgrowth, is associated with neonatal lethal Costello syndrome due to the HRAS p.Gly12Val mutation. Clin Dysmorphol 2019; 28:71-3.

6. Kuniba H, Pooh RK, Sasaki K, Shimokawa O, Harada N, Kondoh T, et al. Prenatal diagnosis of Costello syndrome using $3 \mathrm{D}$ ultrasonography amniocentesis confirmation of the rare HRAS mutation G12D. Am J Med Genet A 2009;149A:785-7.
7. Myers A, Bernstein JA, Brennan ML, Curry C, Esplin ED, Fisher $J$, et al. Perinatal features of the RASopathies: Noonan syndrome, cardiofaciocutaneous syndrome and Costello syndrome. Am J Med Genet A 2014;164A:2814-21.

8. Uemura R, Tachibana D, Kurihara Y, Pooh RK, Aoki Y, Koyama M. Prenatal findings of hypertrophic cardiomyopathy in a severe case of Costello syndrome. Ultrasound Obstet Gynecol 2016;48:799-800.

9. Burkitt-Wright EM, Bradley L, Shorto J, McConnell VP, Gannon C, Firth HV, et al. Neonatal lethal Costello syndrome and unusual dinucleotide deletion/Insertion mutations in HRAS predicting p.Gly12Val. Am J Med Genet A 2012;158A:110210.

10. van der Burgt I, Kupsky W, Stassou S, Nadroo A, Barroso C, Diem A, et al. Myopathy caused by HRAS germline mutations: implications for disturbed myogenic differentiation in the presence of constitutive HRas activation. J Med Genet 2007; 44:459-62.

11. Hague J, Hackett G, Acerini C, Park SM. Prenatal genetic diagnosis of Costello syndrome in a male fetus with recurrent HRAS mutation p.Gly12Ser. Prenat Diagn 2017;37:409-11.

12. Fernandez-Medarde A, Santos E. Ras in cancer and developmental diseases. Genes Cancer 2011;2:344-58.

13. Bertola D, Buscarilli M, Stabley DL, Baker L, Doyle D, Bartholomew DW, et al. Phenotypic spectrum of Costello syndrome individuals harboring the rare HRAS mutation p.Gly13Asp. Am J Med Genet A 2017;173:1309-18. 